PROCEEDINGS OF THE

AMERICAN MATHEMATICAL SOCIETY

Volume 126, Number 5, May 1998, Pages 1287-1291

S 0002-9939(98)04382-2

\title{
CONTINUITY OF K-THEORY: AN EXAMPLE IN EQUAL CHARACTERISTICS
}

\author{
BJØRN IAN DUNDAS
}

(Communicated by Thomas Goodwillie)

\begin{abstract}
If $k$ is a perfect field of characteristic $p>0$, we show that the Quillen K-groups $K_{i}(k[[t]])$ are uniquely $p$-divisible for $i=2,3$. In fact, the Milnor K-groups $K_{n}^{M}(k((t)))$ are uniquely $p$-divisible for all $n>1$. This implies that $K(A) \rightarrow \operatorname{holim}_{\overleftarrow{n}} K\left(A / \mathfrak{m}^{n}\right)$ is 4-connected after profinite completion for $A$ a complete discrete valuation ring with perfect residue field.
\end{abstract}

Let $A$ be a complete discrete valuation ring with maximal ideal $\mathfrak{m}$. Let

$$
K^{t o p}(A)=\underset{n}{\operatorname{holim}} K\left(A / \mathfrak{m}^{n}\right)
$$

We say that K-theory is continuous (at $A$ ) if it commutes with the (inverse) limit, in the sense that

$$
K(A)^{\wedge} \rightarrow K^{t o p}(A)^{\wedge}
$$

is an equivalence, where $X \rightarrow X^{\wedge}$ denotes profinite completion.

This question of continuity has acquired new relevance since the fibers of

$$
K\left(A / \mathfrak{m}^{n}\right)^{\wedge} \rightarrow K(A / \mathfrak{m})^{\wedge}
$$

are now better understood, and have been shown by McCarthy $[\mathrm{Mc}]$ to agree with the corresponding fibers in topological cyclic homology. Hence we are in a position where we sometimes can calculate $K^{\text {top }}(A)$.

One situation where we have an affirmative answer is the theorem of Suslin and Panin $[\mathrm{Su}],[\mathrm{P}]$, which says that if $A$ is a Henselian discrete valuation ring with maximal ideal $\mathfrak{m}$, then

$$
K(A)^{\widehat{\ell}} \rightarrow \underset{\bar{n}}{\operatorname{holim}} K\left(A / \mathfrak{m}^{n}\right) \hat{\ell}
$$

is an equivalence for all primes $\ell$ different from the characteristic of (the field of fractions of) $A$. So, if $A$ is of characteristic zero, then K-theory is continuous at $A$.

This theorem was used critically in Bökstedt and Madsen's calculation [BM] of the K-theory of the $p$-adic integers in order to get the correspondence with topological cyclic homology (here the situation was a bit special, as a similar statement holds for $T C$ ).

Received by the editors October 17, 1996.

1991 Mathematics Subject Classification. Primary 11S70; Secondary 13J05, 19D45, 19D50.

Key words and phrases. Continuity of K-theory, complete discrete valuation ring, ring of formal power series, Milnor K-theory.

The author was supported by the Danish research academy.

(C)1998 American Mathematical Society 
The limiting condition in Suslin/Panin's result is that $\ell$ has to be different from the characteristic of $A$. If $\ell \neq \operatorname{char}(A / \mathfrak{m})$ then a result of Gabber [Ga] tells us furthermore that $K(A) \hat{\ell} \rightarrow K\left(A / \mathfrak{m}^{n}\right) \hat{\ell} \rightarrow K(A / \mathfrak{m}) \hat{\ell}$ are equivalences, and so the situation is really rather degenerate. However, at the characteristic of $A$, K-theory is largely unknown, except for some rather old results in dimensions $\leq 2$.

In dimension 2, much insight can be deduced from a generators-and-relations presentation of $K_{2}(A)$ (e.g. [DS]). It is the hope that a presentation of $K(A)$ as in [D] can shed light on the general situation. In this paper we choose a different approach, in that we try to study the lower K-groups directly by means of Milnor K-theory, and then use Merkur'ev and Suslin's results [MS] to get information about the $\mathrm{K}$-groups themselves.

We take results showing that $K(A)^{\wedge} \rightarrow K^{\operatorname{top}}(A)^{\wedge}$ is somewhat connected for evidence that $K(A)$ may be continuous.

In this note we shall prove

Theorem 1. If $A$ is a complete discrete valuation ring with perfect residue field, then

$$
K(A)^{\wedge} \rightarrow K^{t o p}(A)^{\wedge}
$$

is 4-connected.

Note added in proof (Aug. 26, 1997). It appears that Thomas Geisser has shown that $K_{i}^{i n d}(F)$ is uniquely $p$-divisible for any field $F$ of characteristic $p>0$. Using a slight modification of lemma 6 , the observations in this note then show that $K(A)^{\wedge} \rightarrow K^{t o p}(A)^{\wedge}$ is an equivalence for the rings in theorem 1.

Remark 2. Note that our definition of $K_{i}^{t o p}(A)=\pi_{i} K^{\text {top }}(A)$ differs slightly from, e.g., the notion in $[\mathrm{W}]$, as we are using the homotopy limit. This is connected to the inverse limit of the homotopy groups by the short exact sequence

$$
0 \rightarrow \lim _{\leftarrow}{ }^{(1)} K_{i+1}\left(A / \mathfrak{m}^{n}\right) \rightarrow K_{i}^{\text {top }}(A) \rightarrow \lim _{\leftarrow} K_{i}\left(A / \mathfrak{m}^{n}\right) \rightarrow 0 .
$$

We will be using completion of spectra as in [B]. Note that completions commute with homotopy inverse limits. Recall that if $X$ is a spectrum, then there is an exact sequence

$$
0 \rightarrow \operatorname{Ext}\left(\mathbf{Z}[1 / p] / \mathbf{Z}, \pi_{n} X\right) \rightarrow \pi_{n}\left(X_{p}\right) \rightarrow \operatorname{Hom}\left(\mathbf{Z}[1 / p] / \mathbf{Z}, \pi_{n-1} X\right) \rightarrow 0 .
$$

Remark 3. The reason we have to profinitely complete everything before we ask for an equivalence, is that $K(A) \rightarrow K^{t o p}(A)$ is certainly not an equivalence integrally.

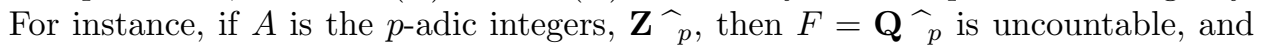
hence by $[\mathrm{M}, 11.10] K_{2}(F)$ is uncountable. By the localization sequence

$$
0=K_{2}\left(\mathbf{F}_{p}\right) \rightarrow K_{2}(A) \rightarrow K_{2}(F) \rightarrow K_{1}\left(\mathbf{F}_{p}\right) \rightarrow 0
$$

we get that $K_{2}(A)$ is uncountable too. But, as each $K_{3}\left(\mathbf{Z} / p^{k} \mathbf{Z}\right)$ is a finite group, we get that the $\lim _{\leftarrow}{ }^{(1)}$ term vanishes, and $K_{2}^{t o p}(A) \cong \lim _{\leftarrow} K_{2}\left(\mathbf{Z} / p^{k} \mathbf{Z}\right)$, which by $[\mathrm{M}, \mathrm{p} .180]$ and $[\mathrm{W}]$ is trivial.

A similar consideration rules out integral continuity for the case of equal characteristics too.

Proof of theorem 1. Let $F$ be the field of fraction of $A$, let $k$ be the residue field, and let $p$ be the characteristic of $k . F$ has characteristic either zero or $p$. The characteristic zero part is taken care of by the Suslin/Panin result mentioned above. 
The only remaining piece is the case where $\operatorname{char}(F)=\operatorname{char}(k)=p>0$. Then $A$ must be isomorphic to the ring of formal power series $k[[t]]$ [Se, II, 4.2]. This is stated separately as proposition 7 below, and the proof will occupy the rest of the paper.

First we prove some useful lemmas regarding the $p$-divisibility of the groups $K_{i}(A)$. This is important in this context, since we have by [He] that $\pi_{i} K^{\text {top }}(A)^{\widehat{\gamma}}=$ 0 for $i>1$.

Lemma 4. Let $A=k[[t]]$ be the ring of formal power series in a perfect field $k$ of characteristic $p>0$, and let $F=k((t))=k[[t]]\left[t^{-1}\right]$ be its field of fractions. Let $n>1$. Then $K_{n}(A)$ is uniquely $p$-divisible if and only if $K_{n}(F)$ is.

Proof. As $k$ is perfect, we get by by $[\mathrm{K}]$ or [Hi] that $K_{n}(k)$ is uniquely $p$-divisible for $n>0$. By $[\mathrm{Ge}, 1.3]$ the localization sequence breaks up into short exact sequences

$$
0 \rightarrow K_{n}(A) \rightarrow K_{n}(F) \rightarrow K_{n-1}(k) \rightarrow 0 .
$$

By hypothesis, multiplication by $p$ is an isomorphism on two of the three groups, and hence also on the third.

For a field $F$, note that $K_{1}(F)$ is the multiplicative group $F^{*}=F-\{0\}$. Milnor $\mathrm{K}$-theory, $K^{*}(F)$, is defined as the graded ring represented as the quotient of the tensor algebra $T_{*}\left(F^{*}\right)=\bigoplus_{n=0}^{\infty}\left(F^{*}\right)^{\otimes n}$ by the homogeneous ideal generated by the elements $x \otimes(1-x) \in F^{*} \otimes F^{*}$. The product in K-theory then defines a map of graded rings $K_{*}^{M}(F) \rightarrow K_{*}(F)$ which is an isomorphism for $*<3$.

Lemma 5. Let $F=k((t))$, where $k$ is a perfect field of characteristic $p$. Then $K_{n}^{M}(F)$ is uniquely p-divisible for $n>1$.

Proof. Since $F$ is of characteristic $p$, the main result of Izboldin [I] gives that $K_{n}^{M}(F)$ has no $p$-torsion, so we just have to show that it is $p$-divisible. We do this by showing that $K_{2}^{M}(F)$ is $p$-divisible. This is enough, for the surjection $\left(F^{*}\right)^{\otimes n} \rightarrow K_{n}^{M}(F)$ factors through $K_{2}^{M}(F) \otimes\left(F^{*}\right)^{\otimes n-2}$, which is $p$-divisible, and so $K_{n}^{M}(F)$ must be $p$-divisible for all $n>1$.

Consider the generator $\{x, y\} \in K_{2}^{M}(F)$, where $x, y \in F^{*}$. We will show that it has a $p$ th root (cf. [M, A.14]). This is clear if there is a $z \in F$ such that $y=z^{p}$, for then $\{x, y\}=\left\{x, z^{p}\right\}=\{x, z\}^{p}$.

On the other hand, suppose $y$ has no $p$ th root in $F$, and consider the inseparable extension $F \subseteq F\left(t^{1 / p}\right)$ of degree $p$. Note that, as $k$ is perfect, the norm map $F\left(t^{1 / p}\right)^{*} \rightarrow F^{*}$ given by $z \mapsto z^{p}$ is surjective $\left(a t^{-N} \prod_{i=0}^{\infty}\left(1-a_{i} t^{i}\right)\right.$ is hit by $\left.a^{1 / p} t^{-\frac{N}{p}} \prod_{i=0}^{\infty}\left(1-a_{i}^{1 / p} t^{\frac{i}{p}}\right)\right)$. In particular, $y$ has a $p$ th root in $F\left(t^{1 / p}\right)$. This means that $F \subset F[z] /\left(z^{p}-y\right) \subseteq F\left(t^{1 / p}\right)$, and since the first inclusion is not an isomorphism, the latter must be, since $p$ is prime.

So, $F \subseteq E=F[z] /\left(z^{p}-y\right)$ is a field extension of degree $p$, and $x$ is in the image of the norm map, and hence [M, 14.3] applies to show that $\{x, y\}$ has a $p$ th root in $K_{2}(F)$.

Lemma 6. If $A=k[[t]]$, where $k$ is perfect of characteristic $p>0$, then $K_{2}(A)$ and $K_{3}(A)$ are uniquely p-divisible.

Proof. We have to show that $K_{2}(F)$ and $K_{3}(F)$ are uniquely $p$-divisible for $F=$ $k((t))$. For $i=2$, note that $K_{2}^{M}(F) \cong K_{2}(F)$ and use the two foregoing lemmas. 
Consider the map $K_{3}^{M}(F) \rightarrow K_{3}(F)$. Call the kernel $K$ and the cokernel $K_{3}^{\text {ind }}(F)$, and we are done if both groups turn out to be uniquely $p$-divisible. By Merkur'ev and Suslin $[\mathrm{MS}], K_{3}^{\text {ind }}(F)$ is uniquely $p$-divisible. Also, $K$ is annihilated by $(3-1) !=2$. If $p=2$, the unique 2-divisibility of $K_{3}^{M}(F)$ implies that $K=0$, and if $p \neq 2$ then $p=2 i+1$ acts as the identity on $K$. Anyhow, $K$ is uniquely p-divisible too.

Proposition 7. Let $k$ be a perfect field. Then

$$
K(k[[t]])^{\wedge} \rightarrow K^{t o p}(k[[t]])^{\wedge}
$$

is 4-connected.

Proof. Let $p$ be the characteristic of $k$. First note that $K_{i}(k[[t]]) \rightarrow K_{i}^{t o p}(k[[t]])$ is an isomorphism if $i<2$, and the groups are without $p$-torsion. If $\ell$ is a prime different from $p$, then $\pi_{i} K(k[[t]]) \hat{\ell} \cong \pi_{i} K\left(k[[t]] / t^{n}\right)_{\hat{\ell}} \cong \pi_{i} K(k) \hat{\ell}$ by [Ga], and so

$$
K(k[[t]]) \hat{\ell} \stackrel{\simeq}{\longrightarrow} K^{t o p}(k[[t]]) \hat{\ell} \stackrel{\simeq}{\longrightarrow} K(k) \widehat{\ell} .
$$

By Hesselholt $[\mathrm{He}], K^{t o p}(k[[t]]) \widehat{p}$ has vanishing homotopy groups in dimension greater than 1 . This is actually only stated for finite fields, but the proof works equally well for perfect fields.

If $G$ is an Abelian group, we have by [BK, VI] that

$$
\operatorname{Ext}(\mathbf{Z}[1 / p] / \mathbf{Z}, G)=0
$$

if and only if $G$ is $p$-divisible, and

$$
\operatorname{Hom}(\mathbf{Z}[1 / p] / \mathbf{Z}, G)=0
$$

if the $p$-torsion elements in $G$ are of bounded order.

So the proposition follows from the exact sequence

$$
0 \rightarrow \operatorname{Ext}\left(\mathbf{Z}[1 / p] / \mathbf{Z}, K_{i}(A)\right) \rightarrow \pi_{i}\left(K(A) \hat{p}_{p}\right) \rightarrow \operatorname{Hom}\left(\mathbf{Z}[1 / p] / \mathbf{Z}, K_{i-1}(A)\right) \rightarrow 0,
$$

the unique $p$-divisibility of $K_{i}(k[[t]])$ for $i=2,3$, and the lack of $p$-torsion in $K_{1}(k[[t]])$.

\section{ACKNOWLEDGMENTS}

It is a pleasure to acknowledge helpful conversations with Steffen Bentzen. This note was written during my stay at Aarhus University, and I am grateful to Ib Madsen for inviting me.

\section{REFERENCES}

[B] A. K. Bousfield, The localization of spectra with respect to homology, Topology 18 (1979), 257-281. MR 80m:55006

[BK] A. K. Bousfield and D. M. Kan, Homotopy limits, completions and localizations, Springer Lecture Notes in Math., vol. 304, 1972. MR 51:1825

[BM] M. Bökstedt and I. Madsen, Algebraic K-theory of local number fields: the unramified case, Ann. of Math. Stud. Princeton University Press 138 (1995), 28-57. MR 97e:19004

[DS] R. K. Dennis and M. R. Stein, $K_{2}$ of discrete valuation rings, Adv. in Math. 18 (1975), 182-238. MR 55:10544

[D] B. I. Dundas, A model for the K-theory of complete extensions, In preparation.

[Ga] O. Gabber, K-theory of Henselian local rings and Henselian pairs, Contemp. Math. 126 (1992), 59-70. MR 93c:19005

[Ge] S. M. Gersten, Some exact sequences in the higher K-theory of rings, Springer Lecture Notes in Math. 341 (1972), 211-243. MR 50:7138 
[He] L. Hesselholt, Topological cyclic homology and local function fields, Aarhus Universitet, Preprint series (31) (December 1993).

[Hi] H. L. Hiller, $\lambda$-rings and algebraic K-theory, J. Pure Appl. Alg. 20 (1981), 241-266. MR 82e: 18016

[I] O. Izhboldin, On p-torsion in $K_{*}^{M}$ for fields of characteristic $p$, Adv. Soviet Math. 4, 129-144. MR 92f: 11165

[K] C. Kratzer, $\lambda$-structure en K-théorie algébrique, Comment. Math. Helv 55 (1980), 233-254. MR 81m: 18011

[Mc] R. McCarthy, Relative algebraic K-theory and topological cyclic homology, To appear in Acta Math.

[MS] A. S. Merkur'ev and A. A. Suslin, The group $K_{3}$ for a field, Math. USSR Izv. 36 (1991), 541-565. MR 91g:19002

[M] J. Milnor, Introduction to algebraic K-theory, Ann. of Math. Stud., vol. 72, Princeton University Press, 1971. MR 50:2304

[P] I. A. Panin, On a theorem of Hurewicz and K-theory of complete discrete valuation rings, Math. USSR Izv. 29 (1987), 81-99. MR 88a:18021

[Se] J. P. Serre, Corps locaux, Actualités scientifiques et industrielles. 1296. Publications de l'Institut de Mathématique de l'Université de Nacango VIII, Hermann, Paris 1968. MR 50:7096

[Su] A. A. Suslin, Algebraic K-theory of fields, Proc. Int. Congr. Math., Berkeley/Calif. 19861 (1987), 222-244. MR 89k:12010

[W] J. B. Wagoner, Delooping the continuous K-theory of a valuation ring, Pacific J. Math. 65 (1976), 533-538. MR 56:3093

Department of Mathematical Sciences, Section Gløshaugen, The Norwegian UniverSity of Science And Technology, N-7034 Trondheim, Norway

E-mail address: dundas@math.ntnu.no 